\title{
Violência conjugal, uso de álcool e sofrimento mental em homens atendidos na atenção primária
}

\section{Conjugal violence, alcohol use and mental suffering of male users of a primary care unity}

\author{
Luciane Carniel Wagner ${ }^{1}$, Juliana Cordeiro Krug², Flávia Koeche ${ }^{3}$
}

\begin{abstract}
RESUMO
Modelo do estudo: Estudo quantitativo, descritivo, transversal, de caráter observacional. Introdução: A violência conjugal é um grave problema de saúde pública, freqüentemente associada ao consumo de álcool, trazendo conseqüências adversas à saúde mental e física das famílias. Seu manejo tem sido um desafio para as políticas sociais, especialmente por causa do medo e do silêncio das vítimas. Em função disso, é bastante interessante a utilização de avaliações de estratégias de resolução de conflito que permitam dimensionar indiretamente (e sutilmente) a violência presente no cotidiano conjugal. Objetivo: Identificar a prevalência de violência conjugal, abuso de álcool e sofrimento mental em homens atendidos (por motivos diversos) em uma unidade básica de saúde de um bairro socialmente carente da periferia de Porto Alegre. Metodologia: Foram avaliados 109 homens, escolhidos ao acaso, utilizando-se como instrumentos de pesquisa a Escala de Avaliação de Táticas de Resolução de Conflitos Conjugais; - CAGE, que acessa o abuso atual e passado de álcool; e o Questionário Geral de Saúde (QSG), que

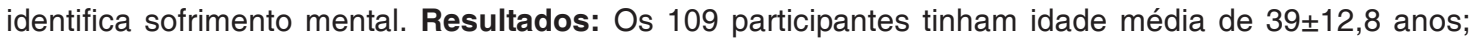
$59,6 \%$ eram brancos; $75,2 \%$ tinham companheira estável; predomínio de baixa escolaridade; $65,1 \%$ exerciam alguma atividade laboral; $80,7 \%$ com renda média mensal de três salários mínimos ou menos. No que tange às táticas de resolução de conflitos conjugais, $24,8 \%$ foram agressivos fisicamente e $84,4 \%$ verbalmente ao menos uma vez no ano anterior à pesquisa. Sofrimento mental considerável foi diagnosticado em $49,5 \%$ dos entrevistados. Com relação ao uso de álcool, $22,9 \%$ responderam afirmativamente para abuso e dependência e outros $24,7 \%$ apresentaram risco de abuso e dependência. Conclusões: 0 estudo aponta para uma elevada prevalência de comportamento conjugal violento, consumo de álcool e sofrimento mental entre os sujeitos avaliados. Estes sujeitos tinham demandas diversas e não necessariamente buscaram o serviço para resolver estes problemas. Os serviços de saúde devem ficar atentos, promovendo estratégias de identificação indiretas, além de medidas de prevenção, que visem combater a violência conjugal e suas conseqüências.
\end{abstract}

Palavras-chave: Conflito Conjugal. Sofrimento Mental. Abuso de Álcool.

1. Médica e Doutora em Psiquiatria, Professora e Pesquisadora do Programa de Pós-Graduação em Reabilitação e Inclusão do Centro Universitário Metodista IPA

2. Terapeuta Ocupacional, Mestranda do Mestrado Profissional Ensino na Saúde FAMED UFRGS, Trabalhadora do Grupo Hospitalar Conceição no CAPS AD III

3. Psicóloga, Bolsista de Pós-Graduação e Mestranda em Biocências e Reabilitação do Centro Universitário Metodista IPA
Correspondencia: Rua: Cel. Joaquim Pedro Salgado, 80 Rio Branco, Cep.: 90420-060, Porto Alegre - RS luciane.wagner@metodistadosul.edu.br

Artigo recebido em 02/09/2013 Aprovado para publicação em 25/04/2014 


\section{Introdução}

A violência conjugal contra a mulher é um grave problema de saúde pública, que gera consequiências adversas à saúde mental e física das vítimas. $\mathrm{O}$ impacto não se restringe à vitima direta da violência, mas aqueles que a testemunham, especialmente os filhos, que se tornam vulneráveis a apresentar problemas de adaptação social, delinqüência e transtornos mentais. ${ }^{1}$ Sua prevalência ao redor do mundo é elevada e pode variar bastante conforme o contexto sociocultural, chegando a $70 \%$ em algumas populações investigadas. ${ }^{2}$ No Brasil, Schraiber e colaboradores encontraram uma prevalência de violência física e/ou sexual de 28,9\% em São Paulo e de 36,9\% na região da Zona da Mata de Pernambuco. ${ }^{3}$

Dentre os problemas associados à violência conjugal, o uso de álcool tem sido apontado como um dos mais frequentes. Um estudo de base populacional, realizado em 143 municípios brasileiros, identificou presença de consumo de álcool em cerca de $40 \%$ dos entrevistados durantes os episódios de violência conjugal. ${ }^{4}$ Fonseca e colaboradores se propuseram a analisar as situações de violência domiciliar ocorridas com o agressor sob efeito do álcool. Investigaram 7.939 domicílios de 108 cidades brasileiras e encontraram que, em 33,5\%, foi relatado histórico de violência domiciliar, sendo $17,1 \%$ com agressores alcoolizados. ${ }^{5}$ Em contextos sociais de grande vulnerabilidade, a freqüência da associação pode ser ainda maior: um estudo realizado em Uganda, com 1.743 mulheres, revelou que 56\% das vítimas de violência conjugal afirmavam que seus companheiros abusavam regularmente de álcool. ${ }^{6}$

A problemática social da violência conjugal ganhou grande visibilidade no Brasil a partir dos anos 80 , quando foram criados os primeiros conselhos municipais e estaduais da mulher, além das delegacias especializadas. A seguir, surgem os abrigos para as vítimas e, em 2003, é promulgada a Lei $\mathrm{n}^{\circ} 10.778$, que estabeleceu a notificação compulsória de casos de violência contra a mulher. A Lei Maria da Penha, promulgada em 2006, institui penas mais severas para os agressores e a criação de juizados especiais de atenção à "violência familiar e doméstica contra a mulher". ${ }^{7}$

A partir do final dos anos noventa também começam a surgir iniciativas de atenção à saúde do homem perpetrador de violência. ${ }^{8}$ Estas medidas nascem da tentativa de ampliar o manejo do problema para além das estratégias punitivas. O sujeito violento frequentemente foi ele próprio vítima de violência. ${ }^{9}$ Suas condições de saúde geral, incluindo sua saúde mental, podem estar comprometidas por comorbidades como transtornos de humor e ansiedade, além do já mencionado abuso de álcool e outras substâncias psicoativas.

Este movimento em direção ao cuidado do homem violento é dificultado pela dinâmica da violência, freqüentemente permeada pela negação do problema, em função do medo e de outros conflitos presentes na vida do casal. ${ }^{10}$ Essa realidade também se estende para a área da pesquisa, em que se observa dificuldade para entrevistar as vítimas, e especialmente, os agressores. Investigações que se refiram diretamente ao homem envolvido em violência conjugal são bem menos freqüentes que investigações com mulheres. Não é incomum que pesquisadores e pesquisados se sintam desconfortáveis e intimidados. ${ }^{2}$

$\mathrm{Na}$ década de setenta, Straus ${ }^{11}$ foi responsável pelo desenvolvimento de um instrumento de avaliação que objetivava investigar as táticas ou estratégias usadas pelas famílias para resolver seus conflitos. Este autor entendia que os conflitos podiam ser resolvidos fundamentalmente de três maneiras: através da argumentação, da agressão verbal ou da agressão física. Estas três dimensões compreendem o instrumento que, indiretamente, permite dimensionar a violência que pode estar presente no cotidiano familiar. Essa medida pode ser bastante útil em situações em que, como já mencionamos, a abordagem direta do fenômeno da violência possa representar uma ameaça para o pesquisador. $^{2}$

Em face do contexto anteriormente exposto, as autoras se propuseram a identificar a prevalência de violência conjugal em homens atendidos (por motivos diversos) em uma unidade básica de saúde de um bairro socialmente carente da periferia de Porto Alegre. Esta avaliação foi feita de modo indireto, acessando-se as táticas de resolução de conflitos conjugais utilizadas por estes homens, através da aplicação do instrumento desenvolvido por Straus. ${ }^{11} \mathrm{O}$ uso/ abuso de álcool e o sofrimento mental destes sujeitos também foram investigados, tendo em vista a importância destas condições na dinâmica da violência.

\section{Metodologia}

Estudo transversal, de caráter observacional, cuja coleta de dados foi realizada entre novembro de 
2006 e abril de 2007, em uma Unidade Básica de Saúde (UBS) de um bairro da periferia da cidade de Porto Alegre. O projeto foi aprovado pelo comitê de ética e pesquisa do Centro Universitário Metodista IPA.

\section{Sujeitos}

Foram incluídos homens entre 18 e 65 anos de idade, que buscassem a atenção primária de saúde por motivos diversos, que aceitassem participar do estudo e conseguissem compreender e responder os instrumentos utilizados no mesmo. Foi estimado um tamanho amostral entre 100 e 150 indivíduos, que correspondia a cerca de $10 \%$ da população total de homens atendidos em um período de seis meses, tempo em que transcorreria a pesquisa na unidade de saúde. Dos 120 sujeitos abordados, 109 preencheram os critérios de inclusão e aceitaram participar do estudo.

\section{Instrumentos utilizados}

Escala de Avaliação de Táticas de Resolução de Conflitos Conjugais (The Conflict Tactics Scale Form $\boldsymbol{R}$ - CTS1): Instrumento desenvolvido por Straus (1979) ${ }^{12}$ que avalia como os casais resolvem seus conflitos. Validado no Brasil por Hasselmann \& Reichenheim (2003) ${ }^{13}$, o instrumento apresentou características psicométricas adequadas na adaptação transcultural, apoiando seu uso no cenário brasileiro. Tem sido utilizado com frequiência em nosso contexto, desde então. ${ }^{14,15}$ Possui dezenove itens, divididos em três dimensões ou "táticas" de resolução de conflitos:
1. argumentação (uso de discussão com linguagem moderada e sensata);

2. agressão verbal (uso de insultos e ameaças com intenção de, simbolicamente, machucar e agredir o outro); e

3. agressão física (uso de força física explícita).

É uma escala do tipo Lickert, com respostas que variam de zero a cinco. Na figura 1 podemos ver um exemplo de pergunta em cada uma das dimensões. Para a avaliação dos resultados, os domínios foram considerados separadamente, inclusive para a análise das relações entre variáveis. Tanto no domínio de agressão verbal como física, consideramos que o fato de ter usado pelo menos uma vez ao ano uma das alternativas mencionadas já significava presença de violência. Escores mais altos indicavam maior uso de uma tática (domínio) particular.

The CAGE questionnaire: Constituído por quatro questões, que devem ser respondidas afirmativa ou negativamente, referentes ao acrônimo cutdown, annoyed, guilty e eye-opener, que acessa o abuso atual e passado de álcool ${ }^{16}$ :

1. Alguma vez o senhor sentiu que deveria diminuir a quantidade de bebida alcoólica ou parar de beber?

2. As pessoas o aborrecem porque criticam o seu modo de tomar bebida alcoólica?

3. O senhor se sente chateado consigo mesmo pela maneira como costuma tomar bebidas alcoólicas?

4. Costuma tomar bebidas alcoólicas pela manhã para diminuir o nervosismo ou ressaca?

Abaixo nós expomos uma lista com algumas das maneiras dos casais resolverem seus conflitos e problemas. Quantas vezes, no último ano, você as utilizou com sua companheira:

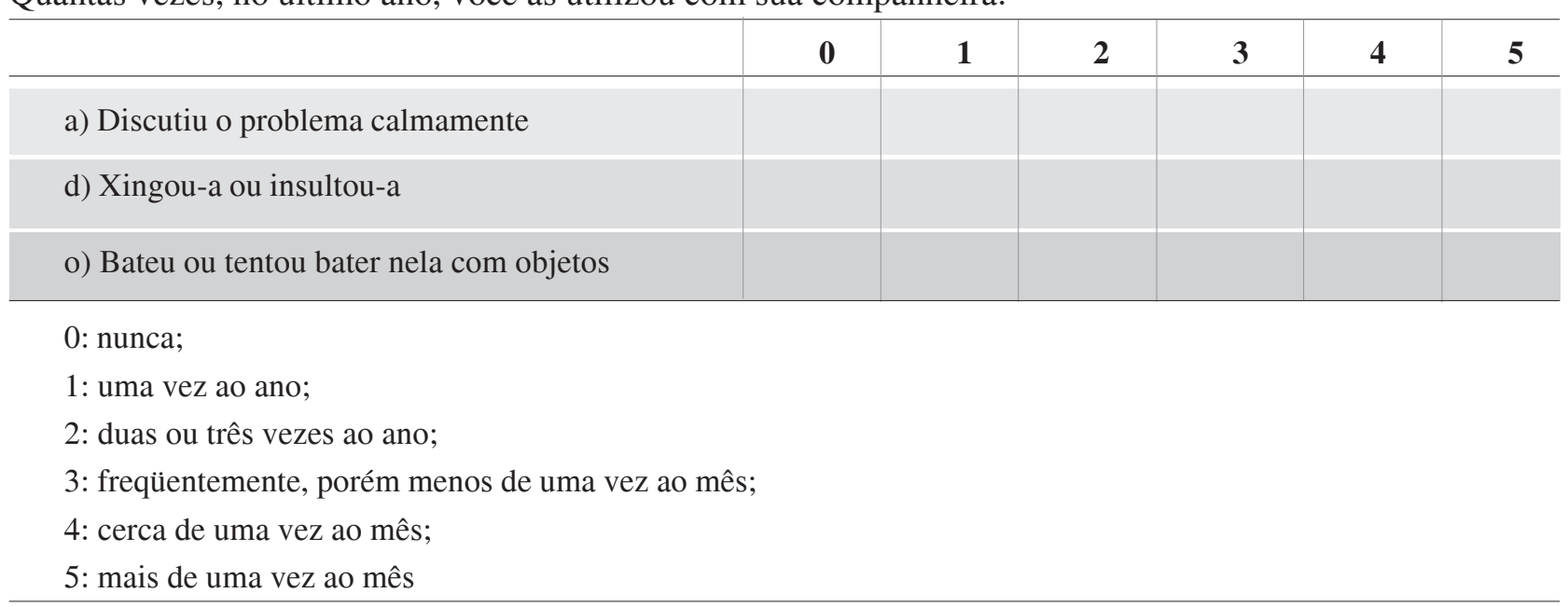

Figura 1. Exemplo de perguntas presentes na Conflict Tactics Scale Form R - CTS1. 
Trata-se de um questionário amplamente utilizado no Brasil, validado por Masur e Monteiro (1983) 17. Duas ou mais questões afirmativas têm uma sensibilidade de $74 \%$ a $76 \%$ e uma especificidade de $91 \%$ a $94 \%$ para o diagnóstico de dependência ou abuso de álcool ${ }^{18,19}$. Além disso, a presença de uma única resposta afirmativa indica risco potencial de vir a desenvolver dependência.

The General Health Questionnaire: Instrumento construído por Goldberg \& Hillier $(1979)^{20}$ para identificar sintomas psiquiátricos tais como depressão ou ansiedade, ocorridos nas duas semanas precedentes ao estudo. Não objetiva o diagnóstico de patologias, mas a triagem de possíveis casos. Originalmente contendo 64 questões, o instrumento apresenta também várias versões reduzidas. No presente estudo, utilizamos uma versão com 12 itens. O instrumento é conhecido no Brasil por Questionário de Saúde Geral (QSG), tendo sido validado por Mari \& Williams (1985). ${ }^{21}$ Para avaliar os resultados, foi usado o ponto de corte 4 , conforme orientação dos criadores da escala, que significa que em 4 das 12 questões houve indicação da presença de sintomas, o que sugeriria que o sujeito avaliado seria um possível caso. Como é uma escala do tipo Lickert com quatro alternativas (Ex: Tem se sentido pouco feliz e deprimido: 1) Não; 2) Como de costume; 3) Mais do que de costume; 4) Muito mais do que de costume), para a análise dos dados a escala foi dicotomizada. No exemplo mencionado anteriormente, se o participante assinalasse as alternativas 1 ou 2, considerava-se ausência daquele sintoma específico; se 3 ou 4, presença.

Questionário sócio-demográfico: os participantes informaram sobre idade, etnia, prática religiosa, nível de educação, situação ocupacional, renda, prática de atividades de lazer e razão porque consulta a Unidade Básica de Saúde. Uma questão sobre a aceitação dos homens entrevistados em receber ajuda para manejar, tratar e controlar o comportamento agressivo por profissionais da atenção primária finalizava a entrevista. O paciente era questionado se concordava ou não em ser ajudado.

\section{Procedimentos}

Dois pesquisadores do curso de terapia ocupacional do Centro Universitário Metodista IPA foram treinados pela pesquisadora responsável (médica-psiquiatra e doutora em psiquiatria) para a aplicação dos instrumentos. Foram feitas duas visitas semanais à UBS durante o período de investigação, em dias e horários regulares. Em cada uma das visitas, eram abordados os cinco primeiros homens que comparecessem à UBS para atendimentos de qualquer natureza e que preenchessem os critérios de inclusão e aceitassem participar da pesquisa. As entrevistas foram realizadas em um consultório médico com privacidade. Os participantes eram esclarecidos sobre objetivos, confidencialidade e liberdade em se retirar da mesma sempre e quando desejassem. O tempo médio de preenchimento dos instrumentos foi de 30 minutos. Dos 120 sujeitos abordados, três não preencheram os critérios de inclusão: dois devido a dificuldades de compreender os instrumentos, um por ter mais de 65 anos; oito não aceitaram participar: quatro alegando falta de tempo, dois por não gostarem de pesquisa, dois não quiseram dar justificativas.

\section{Análise estatística}

Os dados foram descritos como média e desvio padrão para as variáveis quantitativas e frequiências absolutas e relativas para as variáveis qualitativas. Para variáveis sem distribuição normal foram apresentadas a mediana e intervalo interquartis. A comparação entre os grupos foi realizada pelo teste t de Student ou ANOVA para variáveis com distribuição normal e de Mann-Whitney e de Kruskal-Wallis como alternativa para as de distribuição não-normal. As análises de associação entre variáveis categóricas foram feitas pelo Qui-quadrado de Pearson e Teste Exato de Fisher. Os dados foram analisados utilizando-se o programa SPSS versão 13.0. para Windows. O nível de significância adotado foi de $5 \%(\mathrm{p}<0,05)$.

\section{Resultados}

Os 109 participantes tinham idade média de $39 \pm 12,8$ anos; 65 (59,6\%) eram brancos; com predomínio de baixa escolaridade $\left(49,6 \%\right.$ com $1^{\circ}$ grau ou menos, $18,3 \%$ com $2^{\circ}$ grau incompleto e $25,7 \%$ com $2^{\circ}$ grau completo); $68(62,4 \%)$ praticavam alguma religião; $82(75,2 \%)$ tinham companheira (situação conjugal estável há, pelo menos, seis meses); 71 $(65,1 \%)$ exerciam alguma atividade laboral; 81 $(74,3 \%)$ não tinham atividade de lazer; $88(80,7 \%)$ com renda média mensal de três salários mínimos ou menos, sendo que o número máximo de salários mínimos referido pelos participantes foi cinco.

No que tange às táticas utilizadas cotidianamente para a resolução de conflitos conjugais, $27(24,8 \%)$ dos sujeitos entrevistados responderam que foram 
agressivos fisicamente e $92(84,4 \%)$ verbalmente ao menos uma vez no último ano, conforme a CTS1. Na tabela 1 comparamos os sujeitos que foram agressivos fisicamente (que denominamos de "agressores") com os que não o foram ("não agressores"), a fim de investigar alguma possível diferença no que tange a variáveis sociodemográficas relacionadas a vida social, ocupacional, conjugal e à saúde mental entre os dois grupos. Como se pode observar, a única diferença estatisticamente significativa foi no que tange à prática de religião, mais habitual no grupo identificado como agressores.

Quanto à saúde mental destes homens, avaliada pelo QSG, $54(49,5 \%)$ ultrapassaram o ponto de corte estabelecido de mais de quatro questões positivas entre as doze que compõem o questionário, e fo-

Tabela 1: Comparação entre indivíduos agressores e não agressores segundo variáveis selecionadas.

\begin{tabular}{|c|c|c|c|}
\hline Variáveis & Agressores & Não agressores & $p$ \\
\hline \multirow[t]{2}{*}{ Idade $^{a, b}$} & $40,7 \pm 15,4$ & $40,1 \pm 12,7$ & 0,19 \\
\hline & $32[18$ a 64$]$ & $42[19$ a 65$]$ & \\
\hline Com companheira ${ }^{c}$ & $19(70,4)$ & $63(76,8)$ & 0,33 \\
\hline \multirow[t]{2}{*}{ Relacionamentos prévios $\mathrm{a}, \mathrm{b}$} & $2,26 \pm 3,7$ & $3,04 \pm 3,5$ & 0,92 \\
\hline & $2[0$ a 20$]$ & $3[0$ a 13$]$ & \\
\hline Escolaridade $^{c}$ & & & 0,88 \\
\hline $1^{\circ}$ grau incompleto & $9(33,3)$ & $29(35,4)$ & \\
\hline Até $2^{\circ}$ grau completo & $10(37,0)$ & $26(31,7)$ & \\
\hline $2^{\circ}$ grau completo e acima & $8(29,6)$ & $27(32,9)$ & \\
\hline Cor branca ${ }^{c}$ & $15(55,6)$ & $50(61,0)$ & 0,79 \\
\hline \multirow[t]{2}{*}{$\mathrm{QSG}^{\mathrm{a}, \mathrm{b}}$} & $5,7 \pm 3,3$ & $4,6 \pm 2,8$ & 0,18 \\
\hline & 5 [0 a 12] & $4[0$ a 12$]$ & \\
\hline \multirow[t]{2}{*}{$\mathrm{CAGE}^{\mathrm{a}, \mathrm{b}}$} & $1,2 \pm 1,2$ & $0,7 \pm 1,0$ & 0,35 \\
\hline & $1[0$ a 4$]$ & 0 [0 a 4$]$ & \\
\hline Renda $^{c}$ & & & 0,52 \\
\hline Entre 0 e 2 salários mínimos & $44(53,7)$ & $14(51,9)$ & \\
\hline Entre 3 e 5 salários mínimos & $38(46,3)$ & $13(48,1)$ & \\
\hline Tem atividade laboral $^{c}$ & $17(63,0)$ & $54(65,9)$ & 0,82 \\
\hline Pratica atividade religiosa ${ }^{c}$ & $21(77,8)$ & $47(57,3)$ & $0,04 *$ \\
\hline Tem atividade de lazer $^{c}$ & $7(25,6)$ & $21(25,9)$ & 0,58 \\
\hline Total (sujeitos) $^{c}$ & $27(24,8 \%)$ & $82(75,2 \%)$ & \\
\hline
\end{tabular}

Os dados são apresentados como ${ }^{\mathbf{a}}$ média $\pm D P,{ }^{\mathbf{b}}$ mediana [mínimo a máximo], ${ }^{\mathbf{c}}$ contagem (percentual). QSG: Questionário de Saúde Geral. CAGE: The CAGE questionnaire. Número total de sujeitos entrevistados: 109. Com companheira: situação conjugal - há pelo menos seis meses com companheira. Relacionamentos prévios: número de relacionamentos prévios com duração maior que seis meses. ${ }^{*} \mathrm{p}<0,05$ 
ram diagnosticados como caso. Ou seja, quase metade da população entrevistada apresentava sintomas psiquiátricos sugestivos de sofrimento mental nas duas últimas semanas que antecederam a pesquisa. Na tabela 2, pode-se examinar o percentual de sujeitos entrevistados $(\mathrm{N}=109)$ que apresentou sintomas em cada uma das 12 questões do QSG. Os resultados apontam que os problemas mais prevalentes dos participantes são a perda de sono devido a preocupações, a sensação de não superar dificuldades, sentir-se pouco feliz ou deprimido e pouca confiança em si mesmo.

Com relação ao uso de álcool, 25 indivíduos $(22,9 \%)$ responderam afirmativamente para abuso e dependência de álcool (duas ou mais respostas positivas no $C A G E)$ e outros $27(24,7 \%)$ apresentaram risco de abuso e dependência de álcool (uma resposta positiva no $C A G E)$.

\section{Discussão}

O estudo aponta para uma elevada prevalência de comportamento conjugal violento, consumo de álcool e sofrimento mental entre os sujeitos avaliados. Constatamos que quase um terço destes sujeitos re- solveu suas desavenças com a companheira utilizando a agressão física como recurso, pelo menos uma vez no último ano. Também verificamos que a maioria destes sujeitos, ou seja, 84,4\% ( $n=92)$, foi agressivo verbalmente com a companheira quando precisou resolver seus conflitos.

A presença de conflito nas relações sociais e especialmente nas relações conjugais é algo natural e, inclusive, desejável. Alguns autores defendem que sem a presença de conflito nas instituições, nas universidades, nas famílias, estas entrariam em colapso. $12 \mathrm{O}$ conflito, portanto, pode ser entendido como elemento indispensável para a evolução e o desenvolvimento social. O problema é como as pessoas o resolvem. Esta é, sem dúvida, a problemática que emerge do presente estudo: porque razão uma parcela considerável dos sujeitos participantes utiliza a agressão física e muitos mais a agressão verbal como estratégia de resolução de suas discordâncias cotidianas?

Straus (2005) ${ }^{\mathbf{1 2}}$ aponta que a hostilidade se faz mais presente justamente quando os conflitos de interesse entre os sujeitos é negado. A negação do conflito traduz-se pela impossibilidade de resolvê-lo de forma mais madura, refletida, negociada. A impulsi-

Tabela 2. Percentagem dos sujeitos entrevistados (provenientes de uma UBS) apresentando sintomas em cada uma das 12 questões do QSG.

\begin{tabular}{ll}
\hline QSG & $\mathbf{N}(\boldsymbol{\%})$ \\
\hline 1. Não tem podido concentrar-se no que faz & $35(32,1)$ \\
2. Suas preocupações o fazem perder sono & $84(77,1)$ \\
3. Sente-se inútil & $19(17,4)$ \\
4. Incapaz de tomar decisões & $18(16,5)$ \\
5. Sente-se agoniado & $79(72,5)$ \\
6. Tem sensação de não superar dificuldades & $70(64,2)$ \\
7. Incapaz de desfrutar de atividades & $37(33,9)$ \\
8. Incapaz de enfrentar problemas & $27(24,8)$ \\
9. Tem se sentido pouco feliz e deprimido & $60(55,0)$ \\
10. Tem perdido confiança em si mesmo & $51(46,8)$ \\
11. Tem pensado que não serve para nada & $32(29,4)$ \\
12. Não se sente feliz & $22(20,2)$ \\
\hline
\end{tabular}

QSG: Questionário de Saúde Geral. UBS: Unidade Básica de Saúde. Total de sujeitos entrevistados: 109. 
vidade que resulta em agressão é, de fato, uma maneira de "não-pensar" a relação e seus problemas. Seguramente esta é também uma questão cultural, em que as diferenças de gênero podem corresponder a representações sociais de controle por um lado e subordinação por outro.

Outro ponto importante emerge do fato da população investigada proceder de um bairro pobre, com renda média mensal abaixo de três salários mínimos. Um estudo multicêntrico da Organização Mundial da Saúde sobre saúde da mulher e violência doméstica, que investigou cerca de 24.000 mulheres em dez países, encontrou uma prevalência de comportamento violento muito maior em contextos sócio-economicamente vulneráveis. ${ }^{2}$ Sem dúvida, um cotidiano dominado pela precariedade favorece a manutenção de um senso de injustiça social, que não estimula em nada à negociação nas relações, incluindo as conjugais.

A vulnerabilidade social, no entanto, não é um fator exclusivo, ou que aponte uma demarcação estrita de risco, quando se trata da escolha da agressão como tática de resolução de conflitos. Em todos os estratos sociais podemos encontrar violência, embora sabidamente seja mais difícil, eventualmente até impossível, conseguir realizar estudos populacionais que incluam pessoas com alta renda, especialmente no nosso meio. Um estudo feito com 149 casais de nível socioeconômico médio, residentes na capital e no interior do Rio Grande do Sul, utilizando a Escala de Conflito Conjugal, encontrou que, embora preponderassem as formas de resolução em discussões com calma, situações de agressão verbal foram reportadas como já tendo ocorrido em 75,8\% dos casais e episódios de agressão física ou arremesso de objetos já haviam ocorrido em 7,3\% dos casais. ${ }^{22}$

A população investigada parece ter características bastante homogêneas no que tange aos aspectos sociodemográficos. Isso talvez explique o fato de não haver diferenças estatisticamente significativas entre os sujeitos agressivos fisicamente e os demais, exceto no que tange à prática de atividade religiosa. Este achado foi inesperado. Nossa hipótese inicial era de que a prática de alguma atividade religiosa pudesse ser protetora, no sentido de ser mais freqüente entre indivíduos que utilizassem mais o argumento e menos a agressão para resolver seus conflitos. Os achados apontaram o contrário. Isso talvez sinalize que o grupo de "agressores" busque algum auxílio silencioso para suas angústias. Problemas metodológicos também não podem ser esquecidos. Estudos contro- lados precisam ser realizados para avaliar esta variável em sua relação com conflitos conjugais.

Outra hipótese não confirmada era de que os sujeitos agressivos fisicamente pudessem ter mais vulnerabilidade psíquica, o que se evidenciaria pela maior prevalência de abuso de álcool e sofrimento mental. Não encontramos esta diferença. Sem dúvida, o fato de o estudo ser transversal limita bastante as considerações sobre causalidade. Os resultados descritivos, não obstante, são preocupantes, pois demonstram uma prevalência expressiva de dependência de álcool e sofrimento psíquico nos participantes como um todo. Identificamos que $22,9 \%$ dos sujeitos atingiram o ponto de corte de duas respostas afirmativas no $C A G E$, configurando screening positivo para abuso ou dependência de álcool, enquanto que outros $24,7 \%$ estavam em risco para abuso ou dependência de álcool por ter respondido positivamente a uma questão do instrumento. Esta prevalência é superior àquela apontada na literatura para populações gerais. Nos Estados Unidos, por exemplo, este problema atinge cerca de $8,5 \%$ das pessoas, sendo mais freqüente em homens. ${ }^{23}$ No Brasil, um estudo populacional de Almeida e Coutinho (1993) ${ }^{24}$ com 1.459 moradores da Ilha do Governador (RJ), encontrou prevalência de dependência de álcool de 3\%. Outro estudo de base populacional, realizado na cidade de Rio Grande (RS), encontrou que 5,5\% de uma amostra de 1.044 indivíduos abusavam de álcool, dos quais $2,5 \%$ eram dependentes. $^{25}$

Uma investigação de base populacional recente, realizada na cidade de São Paulo com 5.037 adultos, identificou uma prevalência de transtornos relacionados ao uso de substâncias de 3,6\%.$^{26}$ Os autores deste estudo usaram o Composite International Diagnostic Interview (CIDI), na tentativa de descrever o perfil da população entrevistada no que tange à presença de transtornos mentais. Encontraram que os sujeitos com transtornos relacionados ao uso de substâncias, incluindo o álcool, eram os que padeciam de sintomas mais severos de sofrimento mental. $\mathrm{O}$ alto nível de urbanização da megacidade foi correlacionado a um maior risco para a presença de transtornos de controle dos impulsos, assim como a privação social ao abuso de substâncias.

O fato da população investigada em nosso estudo ser somente de homens pode explicar em parte os achados de maior prevalência de dependência alcoólica. A procedência e vulnerabilidade social dos sujeitos também podem estar implicadas nestes acha- 
dos. ${ }^{27}$ Outros fatores, envolvendo a metodologia do estudo, também podem ter contribuído. De fato, o estudo apresenta limitações relacionadas aos critérios de escolha da amostra, que pode não ser representativa da população da região assistida pela Unidade Básica de Saúde investigada. Corroborando com isso, seguramente existe um viés "natural" de seleção, já que os participantes provêm de um serviço de saúde, onde é mais provável que incorram sujeitos com doenças mentais, incluindo a dependência química. Um estudo multicultural, promovido pela Organização Mundial da Saúde, com 1.888 sujeitos provenientes de serviços de atenção primária da Australia, Bulgaria, Kenya, Mexico, Noruega e Estados Unidos encontrou que $18 \%$ deles apresentavam uma alta freqüência de uso considerado perigoso, enquanto que $23 \%$ tinham apresentado problemas diretamente relacionados ao abuso de álcool no ano anterior à pesquisa. ${ }^{28}$ Estas prevalências, assim como a encontrada em nosso estudo, são bastante superiores às de populações gerais.

O sofrimento psíquico foi altamente prevalente entre os investigados (49,5\% dos sujeitos ultrapassaram o ponto de corte do QSG), superando estatísticas para populações gerais, que variam entre 6 a 20\%. ${ }^{\mathbf{2 9 , 3 0 , 3 1}}$ Algumas questões apontadas como justificativas para os achados relacionados à alta prevalência de abuso de álcool cabem aqui: o fato dos entrevistados serem procedentes de uma unidade básica de saúde talvez seja responsável por uma "seleção" não intencional de casos; além disso, a vulnerabilidade social pode ser particularmente relevante. Uma grande proporção destes sujeitos, inclusive, estava desempregada, o que pode ser considerado um estressor ambiental.

Prevalências tão altas de casos parecem ser mais comuns em populações com alguma particularidade, doença ou situação de risco. Na região da Catalunha, Espanha, um estudo identificou que 27\% de um total de 551 de cuidadores de pessoas com esclerose múltipla apresentavam sofrimento mental. ${ }^{\mathbf{3 2}}$ Prevalência bastante superior foi encontrada em sobreviventes de um terremoto no Irã. ${ }^{33}$ Os pesquisadores avaliaram 916 sujeitos, dos quais 58\% foram considerados casos. As altas prevalências da presente investigação talvez estejam relacionadas a ques- tões de vulnerabilidade social, como o desemprego, a baixa escolarização, a renda precária, que se configuram em condições potenciais de risco para o adoecimento mental. Estas condições de vulnerabilidade se assemelham, em última instância, a verdadeiras "catástrofes" sociais.

Quando olhamos isoladamente as questões do instrumento QSG, observamos que problemas para dormir devido a preocupações, sensação de não superar dificuldades, tristeza e pouca confiança em sim mesmo foram os sintomas mais freqüentes. Estes sujeitos não buscaram o serviço de saúde para resolver ou, pelo menos, tentar encontrar algum auxílio terapêutico para tais problemas. Em sua maioria, estavam na Unidade Básica de Saúde para tratar de questões clínicas não relacionadas à saúde mental ou acompanhavam algum familiar. Dessa forma, trata-se, sem dúvida, de uma demanda "indireta", que permanece não acolhida porque se encontra silenciada. Na gênese dos conflitos conjugais e familiares, não obstante, estas demandas seguramente têm seu lugar de destaque, e seguirão produzido sofrimento e, inclusive, violência.

\section{Considerações Finais}

O presente estudo sugere que, apesar do preconceito e do medo, homens fisicamente agressivos contra suas companheiras, abusadores de álcool e com sofrimento mental freqüentam rotineiramente os serviços de saúde, aonde vão devido a outras demandas. Estes achados, em consonância com pesquisas recentes sobre a elevada prevalência de violência doméstica e abuso de substâncias em nosso meio, reforçam a necessidade de ampliar a reflexão sobre o manejo destas condições. As várias estratégias governamentais de combate à violência doméstica, que vêm sendo implementadas desde a década de oitenta, carecem de avaliações sistemáticas de sua efetividade. Da mesma forma, há que se estudar o impacto que a Política Nacional de Atenção Integral à Saúde do Homem (BRASIL, 2008) ${ }^{\mathbf{3 4}}$ vem exercendo sobre as condições globais de saúde masculina em nosso meio, em particular no que diz respeito ao abuso de álcool e outras substâncias psicoativas, notoriamente associados a situações de violência. 


\begin{abstract}
Study model: Quantitative, descriptive and transversal study. Introduction: Conjugal violence is a serious public health issue that is frequently associated with the consumption of alcohol, creating adverse consequences for the mental and physical health of families. Handling this issue has been a challenge for social policy, especially due to the fear and silence of the victims. Therefore, it is very interesting to evaluate conflict resolution strategies that can indirectly (and subtly) affect violence that occurs in day-today married life. Objective: To identify the prevalence of conjugal violence, alcohol abuse, and mental suffering in men given care (for several reasons) in a basic health clinic located in a vulnerable neighborhood in the outskirts of Porto Alegre. Methodology: 109 men were evaluated, chosen at random, using research tools such as the The Conflict Tactic Scale; the CAGE questionnaire that evaluated current and past alcohol abuse; and the General Health Questionnaire (GHQ), that identifies mental suffering. Results: The 109 participants were, on average, $39 \pm 12.8$ years old; $59.6 \%$ were white; $75.2 \%$ had partner; mostly of low education level; $65.1 \%$ exercised manual labor; $80.7 \%$ had an average monthly income of three minimum wages or less. In regard to the conjugal conflict resolution tactics, $24.8 \%$ were physically aggressive, and $84.4 \%$ were verbally aggressive at least once in the year prior to the research. Considerable mental suffering was diagnosed in $49.5 \%$ of those interviewed. In relation to alcohol use, $22.9 \%$ responded in the affirmative to abuse and dependence and another $24.7 \%$ showed risk of abuse and dependency. Conclusions: The study points to a high prevalence of violent conjugal behavior, alcohol consumption and mental suffering among the evaluated subjects. These subjects had various demands and did not necessarily seek the service to resolve these problems. The health services should be aware and promote indirect identification strategies, as well as prevention measures, that seek to combat conjugal violence and its consequences.
\end{abstract}

Key-words: Marital Conflict. Mental Suffering. Alcohol Abuse.

\section{Referências Bibliográficas}

1. Miranda MPM, De Paula CS, Bordin IA. Violência conjugal física contra a mulher na vida: prevalência e impacto imediato na saúde, trabalho e família. Rev Panam Salud Publica. 2010; 27: $300-8$

2. Ellsberg M, Jansen HAFM, Heise L, Watts $\mathrm{CH}$, Garcia-Moreno C. Intimate partner violence and women's physical and mental health in the WHO multicountry study on women's health and domestic violence: an observational study. Lancet. 2008; 371:1165-72.

3. Schraiber LB, D'Oliveira AFPL, França Jr I, Diniz S, Portella $A P$, Ludermir AB, et al. Prevalência da violência contra a muIher por parceiro íntimo em regiões do Brasil. Rev Saúde Publica. 2007; 41: 797-807.

4. Zaleski M, Pinsky I, Laranjeira R, Ramisetty-Mikler S, Caetano R. Intimate partner violence and alcohol consumption. Rev Saúde Publica. 2010; 44: 53-9.

5. Fonseca AM, Galduroz JCF, Tondowski CS, Noto AR. Padrões de violência domiciliar associada ao uso de álcool no Brasil. Rev Saúde Publica. 2009; 43: 743-9.

6. Tumwesigye NM, Kyomuhendo GB, Greenfield TK, Wanyenze RK. Problem drinking and physical intimate partner violence against women: evidence from a national survey in Uganda. BMC Public Health. 2012; 12: 399.

7. Oliveira KLC, Gomes R. Homens e violência conjugal: uma análise de estudos brasileiros. Ciênc Saúde Coletiva. 2011; 16: 2401-13.

8. Lima DC, Büchele F, Clímaco DA. Homens, gênero e violência contra a mulher. Saúde Soc. 2008; 17: 69-81.

9. Ribeiro WS, Andreoli SB, Ferri CP, Prince M, Mari JJ. Exposure to violence and mental health problems in low and middle-income countries: a literature review. Rev Bras Psiquiatr. 2009; 31(suppl.2): S49-S57.
10. Kronbauer JFD, Meneghel SN. Perfil da violência de gênero perpetrada por companheiro. Rev Saúde Publica. 2005; 39: 695-701.

11. Straus MA. Measuring Intrafamily Conflict and Violence: The Conflict Tactics Scales. J Marriage Family. 1979; 41: 75-88.

12. Straus MA. Reflections on Measuring Intrafamiiy Conflict and Violence. In: Bergen RK, Edleson JL, Renzetii CM, Editors. Violence Against Women: Classic Papers. Boston: Pearson Education Cnc., 2005, p. 195-7.

13. Hasselmann MH, Reichenheim ME. Cross-cultural adaptation of the Portuguese version of the Conflict Tactics Scales Form R (CTS-1) used to assess marital violence: semantic and measurement equivalence. Cad Saúde Pública. 2003; 19:1083-93

14. Paim K, Madalena M, Falcke D. Esquemas iniciais desadaptativos na violência conjugal. Rev Bras Ter Cogn. 2012; 8: 31-9.

15. Bolzes SDA, Crepaldi MA, Schmidt B, Vieira ML. Relacionamento Conjugal e Táticas de Resolução de Conflito entre Casais. Actual Psicol. 2013; 27: 71-85.

16. Mayfield D, McLeod G, Hall P. The CAGE questionnaire: validation of new alcoholism screening instrument. Am J Psychiatr. 1974; 131: 1121-3.

17. Masur J, Monteiro MG. Validation of the "CAGE" alcoholism screening test in a Brazilian psychiatric inpatient hospital setting. Braz J Med Biol Res. 1983; 16: 215-8.

18. Beresford TP, Blow FC, Hill E, Singer K, Lucey MR. Comparison of CAGE questionnaire and computer-assisted laboratory profile in screening for covert alcoholism. Lancet. 1990; 336: $482-5$.

19. Buchsbaum DG, Buchanan RG, Centor RM, Schnoll SH, Lawton MJ. Screening for alcohol abuse using CAGE scores and likelihood ratios. Ann Intern Med. 1991; 115: 774-7. 
20. Goldberg DP, Hillier VF. A scaled version of the General Health Questionnaire. Psychol Med. 1979; 9: 139-45.

21. Mari JJ, Williams P. A comparison of the validity of two psychiatric screening questionnaires (QSG-12 and SRQ-20) in Brazil, using Relative Operating Characteristic $(\mathrm{ROC})$ analysis. Psychol Med. 1985; 15: 651-9.

22. Mosmann C, Falcke D. Conflitos conjugais: motivos e frequência. Rev SPAGESP. 2011; 12: 5-16.

23. Falk D, Yi H, Hiller-Sturmhöfel $\mathrm{S}$. An epidemiologic analysis of co-occurring alcohol and drug use and disorders: findings from the National Epidemiologic Survey of Alcohol and Related Conditions (NESARC). Alcohol Res Health. 2008; 31:100-10.

24. Almeida LM, Coutinho ESF. Prevalência de consumo de bebidas alcoólicas e de alcoolismo em uma região metropolitana do Brasil. Rev Saúde Publica. 1993; 27: 23-9.

25. Primo NLNP, Stein AT. Prevalência do abuso e da dependência de álcool em Rio Grande (RS): um estudo transversal de base populacional. Rev Psiquiatr Rio Gd Sul. 2004; 26: 280-6.

26. Andrade AG, Silveira CM. Problemas comportamentais ligados ao uso de álcool. Rev. USP. 2012; 96: 7-22.

27. Murali V, Oyebode F. Poverty, social inequality and mental health. Adv Psychiatr Treat. 2004; 10: 216-24.

28. Saunders JB, Aasland OG, Amundsen A, Grant M. Alcohol consumption and related problems among primary health care patients: WHO Collaborative Project on Early Detection of Persons with Harmful Alcohol Consumption-I. Addiction. 1993; 88: 349-62.
29. Hoeymans N, Garssen AA, Westert GP, Verhaak PFM. Measuring mental health of the Dutch population: a comparison of the GHQ-12 and the MHI-5. Health Qual Life Outcomes. 2004; 2: 23.

30. Mann RE, Cheung JTW, lalomiteanu A, Stoduto G, Chan V, Wickens $\mathrm{CM}$, et al. Estimating prevalence of anxiety and mood disorder in survey data using the GHQ12: Exploration of threshold values. Eur J Psychiatry. 2011; 25: 81-91.

31. Noorbala AA, Bagheri Yazdi SA, Yasamy MT, Mohammad K. Mental health survey of the adult population in Iran. $\mathrm{Br} \mathrm{J}$ Psychiatr. 2004; 184:70-3.

32. Aymerich M, Guillamón I, Jovell AJ. Health-related quality of life assessment in people with multiple sclerosis and their family caregivers. A multicenter study in Catalonia (Southern Europe). Patient Prefer Adherence. 2009; 3: 311-21.

33. Montazeri A, Baradaran H, Omidvari S, Azin SA, Ebadi M, Garmaroudi G, et al. Psychological distress among Bam earthquake survivors in Iran: a population-based study. BMC Public Health. 2005; 5: 4.

34. BRASIL. Ministério da Saúde. Secretaria de Atenção à Saúde. Departamento de Ações Programáticas Estratégicas. Política Nacional de Atenção Integral à Saúde do Homem. Brasília, 2008. 40 p. 